\title{
Relationship Between Blood Pressure and Heart Rate Circadian Rhythms in Normotensive and Hypertensive Subjects
}

\author{
Giulia Silveri ${ }^{1}$, Lorenzo Pascazio ${ }^{2}$, Agostino Accardo ${ }^{1}$ \\ ${ }^{1}$ Department of Engineering and Architecture, University of Trieste, Trieste, Italy \\ ${ }^{2}$ Department of Medicine, Surgery and Health Science, ASUITs, Trieste, Italy
}

\begin{abstract}
This paper focuses on the relationship between blood pressure (BP) and heart rate (HR) during 24 hours in 423 normotensive (NO) and 205 hypertensive (HE) subjects. Although considerable knowledge has been gained about $B P$ and HR signals, their relationship over 24 hours has never been completely described. By using a Holter Blood Pressure Monitor, it was possible to record BP and HR for 24 hours. Systolic, Diastolic and Mean BP in both NO and $H E$ subjects showed four different time intervals presenting well-defined trends The results demonstrated that changes in HR present closely parallel changes in $B P$ with a marked reduction of both signals during nocturnal rest. On the contrary, in the period between 15:30 and 19:30, HR and BP showed an inverse relationship with decreasing heart rate and increasing blood pressure.
\end{abstract}

\section{Introduction}

It is well known that variability signals related to cardiovascular system contain relevant information about the behavior of the autonomic nervous system that acts as a controller of many physiological parameters such as heart rate (HR) and blood pressure (BP) [1-3]. In particular, HR measurements provide significant prognostic information about cardiovascular risks [4]. On the other hand, BP measurements represent a powerful prognostic marker of target organ damage [5].

The analysis achieved on both 4-Chicago epidemiologic surveys [6] and a nationwide Belgian population study [7], carried out on approximately 5000 men and 4000 women, showed that HR progressively increased when systolic blood pressure (SBP) and diastolic blood pressure (DBP) increased, with a stronger direct association for SBP than for DBP values.

Several studies investigated whether the relationship between heart rate and blood pressure was linear [8-12]. In particular, Reed et al. [8] found a linear relationship between HR and both SBP and DBP in the range 70-90 beats/min. Erikssen and Rodahl [9] reported a constant increase in SBP from 40 to 100 beats/min in adults and Schieken et al [10] observed a similar increase in children.

Moreover, some epidemiologic studies shown a direct relationship between $\mathrm{HR}$ and $\mathrm{BP}$ increase in general population, across the age [7][12].

In addition, hypertension (SBP $>140 \mathrm{mmHg}$ and DBP $>90 \mathrm{mmHg}$ [13]) is recognized as a key risk factor for cardiovascular disease mortality and his treatment is associated with a decrease in cardiovascular complications [14]. The ABP-International study found in 7600 untreated patients with hypertension that every 10 bpm of HR increase during nighttime could be associated with $13 \%$ of increased risk of cardiovascular events [15]. Another study in 566 patients with hypertension showed that nighttime HR values above $65 \mathrm{bpm}$ could be associated with the presence of target organ damage [16].

Although considerable knowledge has been gained about the connection between BP and HR, the relationship over 24 hours has never been completely described although it is known that both signals have a circadian rhythm and they can be measured with noninvasive techniques [17].

Several authors highlighted that the HR during sleep presents lower values than during daytime [18-20] and the BP shows a higher level during the daytime than during the night $[21,22]$. Moreover, the day-night difference in the heart rate was positively associated with the day-night differences in office SBP and DBP. Hence, the diurnal variations of heart rate tended to parallel the diurnal variations in blood pressure and to decrease with reducing blood pressure [23].

In order to fill the lack of research about the relationship between BP and HR over 24 hours in this study we examined this link in normotensive and hypertensive subjects.

\section{Methods}

A sample of 628 subjects (261 males and 367 females) was recruited at the Ambulatory of Physiopathology for Elderly of the Geriatric Department (ASUITs, Trieste, 
Italy). The HR and BP behaviour of each participant, without either clinical evidence of hypertension-related complications or clinical or laboratory evidence of secondary causes of arterial hypertension or cardiac disease, were examined. All subjects gave their written informed consent.

The subjects were divided in 423 normotensive (NO) with $\mathrm{SBP} \leq 140 \mathrm{mmHg}$ and $\mathrm{DBP} \leq 90 \mathrm{mmHg}$, and 205 hypertensive (HE), with $\mathrm{SBP}>140 \mathrm{mmHg}$ and $\mathrm{DBP}>90$ $\mathrm{mmHg}$ [6]. These pressures were measured in office condition, as required by [6].

To record BP and HR parameters, a Holter Blood Pressure Monitor, (Mobil-O-Graph ${ }^{\circledR}$ NG, IEM gmbh Stolberg, Germany), based on oscillometric technique, was used over 24 hours. The acquisition rate was of 15 minutes throughout the day and of 30 minutes throughout the night. Systolic readings greater than $260 \mathrm{mmHg}$ or less than $70 \mathrm{mmHg}$, as well as, diastolic readings greater than $150 \mathrm{mmHg}$ or less than $40 \mathrm{mmHg}$ were automatically discarded. The BP and HR values of the different subjects were aligned using common start time (10:00) since recordings could start at different times of the day (between 8:00 and 11:00). The circadian behaviour of the mean values of SBP, DBP and Mean BP (i.e. $[2 * \mathrm{DBP}+\mathrm{SBP}] / 3$ ) among the subjects was separately examined for normotensive and hypertensive subjects.

In order to compare the HR values in the 24 hours in the two groups, the Wilcoxon signed rank sum test was used.

\section{Results}

Figure 1 (left panels) shows that SBP, Mean BP and DBP decrease between 9:30 and 15:00 (blue line), then they present a moderate increase between 15:00 and 19:30 (red line) and a deeper reduction during night-time from 19:30 till about 2:00 (black line). An about constant behaviour is present between 2:00 and 5:30 (green line) followed by a morning increase between 5:30 and 9:30. A similar trend during the 24 hours is present both in NO and HE subjects with an about constant difference of 20 $\mathrm{mmHg}$ during all the 24 hours.

Figure 2 illustrates the circadian rhythm of HR presenting slow fluctuating decrease during the day from about 9:30 until 19:30 and a quicker decrease from 19:30 till 2:00. From 2:00 to 6:00 the HR was about constant followed by a quick increase during the morning from about 6:00 till 930 .
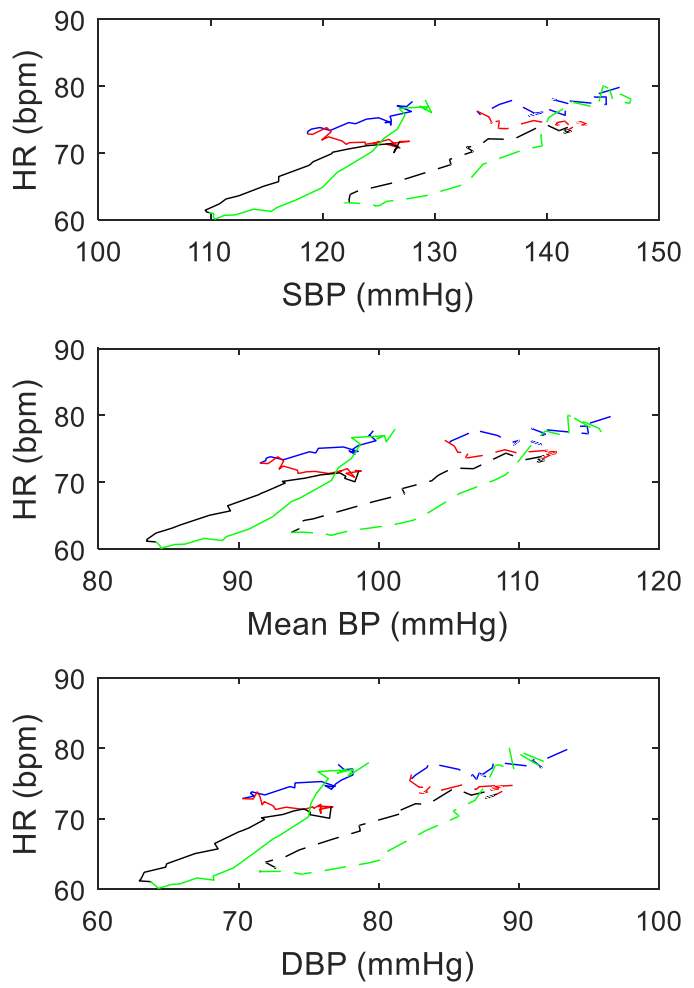

Figure 1. Circadian rhythms of SBP, Mean BP, DBP (left panels) and relationships between HR and SBP, Mean BP and DBP (right panels) in Normotensive (solid line) and Hypertensive subjects (dashed line). Blue line: 9:30 - 15:00; red line: 15:00 - 19:30; black line: 19:30 - 2:30; green line: 2:30 - 9:30. 


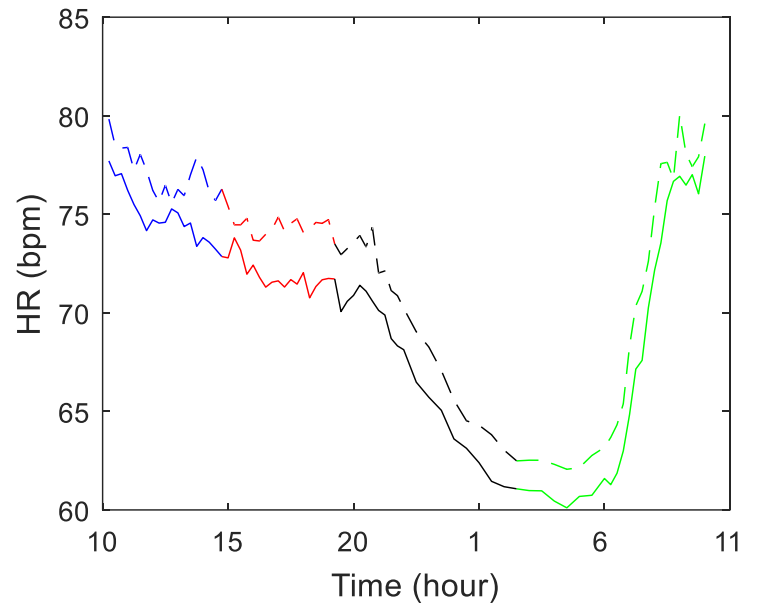

Figure 2. Circadian rhythm of HR in Normotensive (solid line) and Hypertensive (dashed line) subjects. Blue line: 9:30 - 15:00; red line: 15:00 - 19:30; black line: 19:30 - 2:30; green line: 2:30 - 9:30.

In this trend, the HR values were significantly higher $(\mathrm{p}<0.001)$ in $\mathrm{HE}$ than in NO during all the 24 hours with a difference between $\mathrm{HE}$ and $\mathrm{NO}$ of about $2.3 \pm 0.8 \mathrm{bpm}$.

The relationships between SBP, Mean BP, DBP and HR circadian rhythms in NO and HE subjects are showed in Figure1, right panels. Between 9:30 to 15:00 (blue line), the HR and SBP decrease progressively while during postprandial hours, between 15:00 to 19:30 (red line), SBP increases and HR still decreases. During the first part of the night (from 19:30 to 2:30), HR and SBP show a marked reduction with the presence of hysteresis in respect of the quick increase of both SBP and HR values during the successive morning period, from 2:30 to 9:30 (green line). The hypertensive subjects present a similar behaviour during the 24 hours with obviously greater pressure values. Finally, the relationships between Mean BP, DBP and HR are similar to that of the SBP versus HR.

\section{Discussion}

HR and BP are signals investigated in several different clinical and physiological conditions and a few of paper studied the relationship between them although how this relationship changes during 24 hours has not been examined yet. In this paper, we studied the circadian relationship between blood pressure and heart rate rhythms in normotensive and hypertensive subjects.

The circadian BP and HR changes are affected by a variety of external factors as well as of internal factors such as autonomic nervous system tone, vasoactive hormones, hematologic and renal variables and they are markers of target organ damage and cardiovascular risk [4-5].
The results show a characteristic circadian variation of BP and HR as well as a specific relationship between these two parameters (Figs.1 and 2). The circadian behaviour is similar both in NO and HE subjects

The changes in heart rate variability present closely parallel changes in blood pressure (SBP, DPB and Mean $\mathrm{BP})$ with a marked reduction during nocturnal rest. Our results confirm previously outcomes concerning generally lower values of HR and BP during the night than during the day [18-22] as well as a direct association between HR and SBP and DBP increasing [6-12] at least in period in which HR increased (2:30-9:30). Moreover, a direct link was also found between BP and HR decreasing in the 9:30-15:00 and 19:30-2:30 periods. On the contrary, in the interval between 15:30 and 19:30, HR and BP showed an inverse relationship with decreasing heart rate and increasing blood pressure (Fig.1, left panels). This latter inverse behaviour was not yet reported in the literature and should be better analysed in the future.

Furthermore, a true linear relationship between HR and $\mathrm{BP}$ values was found only during the night decrease of BP (19:30-2:30), partially confirming previously literature results [8-10]. In the other three periods of the day, the relationship changed drastically and was mostly not linear.

The study of BP and HR behaviours as well as they relationship in NO and $\mathrm{HE}$ subjects during 24 hours indicated that the circadian changes are very similar in both groups suggesting that circadian cardiovascular modulation may not be impaired by hypertension.

\section{Conclusion}

In this study, the differences during 24 hours of BP and HR parameters, as well as, their circadian relationship in normotensive and hypertensive subjects were examined. Besides confirmation of previously results concerning similarities between BP and HR circadian behaviours, our study also highlighted some new findings thanks to the more detailed analysis carried out during the 24 hours. In particular, an inverse association between BP and HR was found in a specific period of the day, but not yet reported in the literature. Further studies to explain this particular result are needed.

\section{Acknowledgements}

Work partially supported by the Master in Clinical Engineering, University of Trieste.

\section{References}

[1] De Boer RW, Karemaker JM. Hemodynamic fluctuations and baroreflex sensitivity in humans: a beat to beat model. American Journal of Physiology 1987;243:680-689.

[2] Johansen CD, Olsen RH. Resting night-time, and $24 \mathrm{~h}$ heart 
rate as markers of cardiovascular risk in middleaged and elderly men and women with no apparent heart disease. European Heart Journal 2013;34(23):1732-1739.

[3] Guyenet PG. The sympathetic control of blood pressure. Natur Reviews Neuroscience 2006;7:335-346.

[4] Palatini P, Julius S. Heart rate and cardiovascular risk Journal of Hypertension 1997;15:3-17.

[5] Diaz KM, Tanner RM. Visit-to-visit variability of blood pressure and cardiovascular disease and all-cause mortality; a systematic review and meta-analysis. Hypertension 2012;60:625-630.

[6] Stamler J, Berkson DM. Relationship of multiple variables to blood pressure-findings from four Chicago epidemiologic studies. In: Paul O, editor. Epidemiology and Control of Hypertension. Miami: Symposia Specialists 1975;1:307-352.

[7] Zhang J, Kesteloot H. Anthropometric, lifestyle and metabolic determinants of resting heart rate. A population study. European Heart Journal 1999;20:103-110.

[8] Reed D, McGee D. Biological and social correlates of blood pressure among Japanese men in Hawaii. Hypertension 1982;4:406-414.

[9] Erikssen J, Rodahl K. Resting heart rate in apparently healthy middleaged men. European Journal Applied Physiology 1979;42:61-69.

[10] Schieken RM, Clarke WR. Left ventricular hypertrophy in children with blood pressures in the upper quintile of the distribution The Muscatine study. Hypertension 1981;3:669-675.

[12] Hansen TW, Thijs L. Prognostic value of ambulatory heart rate revisited in 6928 subjects from 6 populations. Hypertension 2008;52:229-35.

[13] 2003 World Health Organization (WHO)/International Society of Hypertension (ISH) statement on management of hypertension. Journal of Hypertension 2003;21:1983-1992.

[14] Palatini P, Saladini F. Low night-time heart rate is longitudinally associated with lower augmentation index and central systolic blood pressure in hypertension. European Journal of Applied Physiology 2018;118(3):543550.

[15] Palatini P, Reboldi G. Predictive value of nighttime heart rate for cardiovascular events in hypertension. The ABPInternational study. International Journal of Cardiology 2013;168(2):1490-5.

[16] Facila L, Pallares V. Twenty-four-hour ambulatory heart rate and organ damage in primary hypertension. Blood Press 2010;19(2):104-9.

[17] Mancia G, Parati G. Ambulatory blood pressure monitoring and organ damage. Hypertension 2000;36:894-900.

[18] Palatini P, Penzo M. Clinical relevance of nighttime blood pressure and of daytime blood pressure variability. Arch Internal Medicine 1992;152:1855-1860.

[19] Millar-Craig MW, Bisop CN. Circadian variation in blood pressure. Lancet 1978;1:795-797.

[20] Mancia G, Ferrari A. Blood pressure and heart rate variabilities in normotensive and hypertensive human beings. Circulation Research 1983;53:96-104.

[21] Zakopoulos N, Stamatelopoulos S. Effect of hypotensive drugs on the circadian blood pressure pattern in essential hypertension: A comparative study. Cardiovascular Drugs Therapy 1997;11:795-799.

[22] Littler WA, West MJ. The variability of arterial pressure.
American Heart Journal 1978;95:180-186.

[23] Verdecchia P. Prognostic Value of Ambulatory Blood Pressure. Current Evidence and Clinical Implications. Hypertension 2000;35:844-851.

Address for correspondence:

Giulia Silveri

University of Trieste

Via A.Valerio 10, 34127, Trieste, Italy

giulia.silveri@phd.units.it 